\title{
A Unified Approach for Generalizing Some Families of Probability Distributions, with Applications to Reliability Theory
}

\author{
Ahmed. A. Fattah \\ Department of mathematical statistics \\ Institute of Statistical Studies \& Research, Cairo University, Egypt \\ ahmedzi1987@gmail.com
}

\author{
A-hadi N. Ahmed \\ Department of mathematical statistics \\ Institute of Statistical Studies \& Research, Cairo University, Egypt \\ drhadi2010@gmail.com
}

\begin{abstract}
In this paper, we propose a new method for generating families of continuous distributions based on the star-shaped property which grantees the existences of some well know properties for the generated classes and distributions for any non-negative random variables. We refer to the new class as the composed $-G Q$ generator or shortly $(C-G Q)$ generator. We study some mathematical properties of the new family. Some special families and sub-models from the $C-G Q$ generator are discussed. To examine the performance of our new generator and the generated models in fitting several data we use two real sets of data; censored and uncensored then comparing the fitting of a new produced model called composed- Lomax Weibull $(C-L W)$ with some well-known models, which provides the best fit to all of the data. A simulation has been performed to assess the behavior of the maximum likelihood estimates of the parameters under the finite samples.
\end{abstract}

Keywords: Star Shaped, Survival Function, Moment Generating Function, Inversion Method, Order Statistics, Maximum Likelihood Estimation.

\section{Introduction}

In recent years there has been an increased interest in defining new generators for univariate continuous distributions by introducing one or more additional parameter(s) to the baseline distribution. This induction of parameter(s) has been proved useful in exploring tail properties and also for improving the goodness-of-fit of the proposed family and provides great flexibility in modeling data in practices.

Depending on a distribution with $g(x), G(x)$ and $\bar{G}(x)$ as the probability density function (pdf), the cumulative distribution function (cdf) and the survival functions (sf), respectively, a lot of generators have been introduced in the literature based on the pdf or the cdf or also the sf as the base distribution to introduce new classes.

So, in the following context we try to introduce a new method for generating a wide number of classes with known characteristics in the reliability theory.

Definition 1. Let $G$ and $Q$ be two arbitrary continuous cdf's distributions of an absolutely continuous random variable, $G$ be strictly increasing on its support, and $G(0)=$ $Q(0)=0$. Now define a cdf, $F$, out of $G$ and $Q$ (called the composed $-G Q$ family shortly $(C-G Q))$ as follows:

$$
F(x)=G(x \cdot Q(x)), \quad \forall x \geq 0,
$$


and its corresponding pdf is given by

Natural extensions of (1) is

$$
f(x)=g(x \cdot Q(x)) \cdot\{x \cdot q(x)+Q(x)\}
$$

Or

$$
F_{a}(x)=G\left(x \cdot \prod_{i=1}^{n} Q_{i}(x)\right)
$$

$$
F_{b}(x)=G\left(x . \sum_{i=1}^{n} Q_{i}(x)\right)
$$

One can use $F_{a}(x)$ and $F_{b}(x)$ for generating numerous families with a wide number of distributions based on two or more cdf's.

Definition 2. Let $G$ be a continuous cdf distribution of an absolutely continuous random variable and $H($.$) is the cumulative hazard rate function. Now define a cdf, F_{1}$, out of $G$ and $H$ as follows:

$$
F_{1}(x)=G(x \cdot H(x)), \quad \forall x,
$$

and its corresponding pdf is given by

$$
f_{1}(x)=g(x \cdot H(x)) \cdot\{x \cdot h(x)+H(x)\}
$$

Next we show a set of important results in the theoretical reliability hold for our newly introduced family. These results make the family much richer in applications. These results extracted from Barlow and Proschan (1981) and are listed below for convenience.

Let $F$ and $G$ be continuous distributions, $G$ be strictly increasing on its support, and $F(0)=G(0)=0$. Then $F$ is star-shaped with respect to $G$ (written $F<G$ ) if $G^{-1} F(x)$ is star-shaped [that is, $\frac{1}{x} G^{-1} F(x)$ is increasing for $x \geq 0$ ]. Then:

a) $F<_{c} G$ implies $F<_{*} G$ (where $<_{c}$ implies the convex ordering).

b) The relationship $F<_{c} G$ is unaffected by a translation transformation of either $F$ and $G$, assuming the random variables remain non-negative.

c) The relationship $F<G$ may be destroyed by a translation transformation of either $F$ and $G$, assuming the random variables remain non-negative.

d) Let $G(x)=1-e^{-\lambda x}, F$ be a continuous distribution function, with $F(0)=0$. Then $F<_{c} G$ is equivalent to $F$ IFR.

e) Let $G(x)=1-e^{-\lambda x}, F$ be a continuous distribution function, with $F(0)=0$. Then $F<G$ is equivalent to $F$ IFRA.

The Single Crossing Property. Let $F<_{c} G$, then

i) $\bar{F}(x)$ crosses $\bar{G}(\theta x)$ at most one, and from above, as $\mathrm{x}$ increases from 0 to $\infty$, from $\theta>0$.

ii) If, in addition, $F$ and $G$ have the same mean, then a single crossing does occur, and $F$ has smaller variance than $G$.

iii) If we take $G$ to be the exponential distribution, then $F$ must be IFRA by the previous results. 
To this end, we present the following arguments.

Based on Definition 1. we can see that the new generator enjoys the star-shaped property, which means any distribution derived based on the new family enjoys the results form a. to e..

Suppose that $G(x)$ and $Q(x)$ are the cdf's of the exponential distribution and are respectively given by $G(x)=1-e^{-\lambda x}$ and $Q(x)=1-e^{-\beta x}$ (for $x>0$ and $\lambda, \beta>0$ ). Then, a new distribution called composed-exponential exponential $(\mathrm{C}-E E)$, can be derived based on (1), and its cdf is given by

$$
F(x)=G(x \cdot Q(x))=1-e^{-\lambda x \cdot\left[1-e^{-\beta x}\right]}, \quad \lambda, \theta>0, x>0,
$$

while, its corresponding pdf is given by

$$
f(x)=\lambda e^{-\lambda x \cdot\left[1-e^{-\beta x}\right]}\left[1+(\beta x-1) e^{-\beta x}\right] .
$$

Now, we check the existence of the star-shaped property for the new generated model $\mathrm{C}-E E$.

i For any given values of $\lambda, \beta$ and $\theta$, then $\bar{F}(x)$ crosses $\bar{G}(\theta x)$ at most one, and from above, as $x$ increases from 0 to $\infty$, for $\theta>0$.

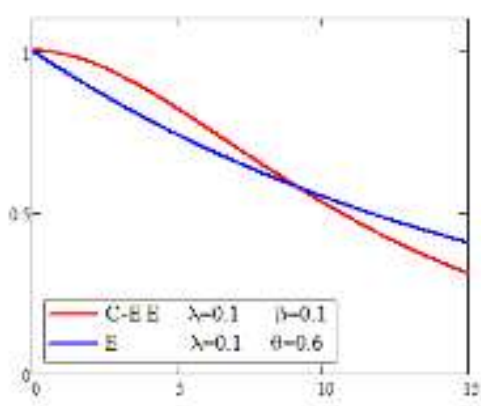

(a)

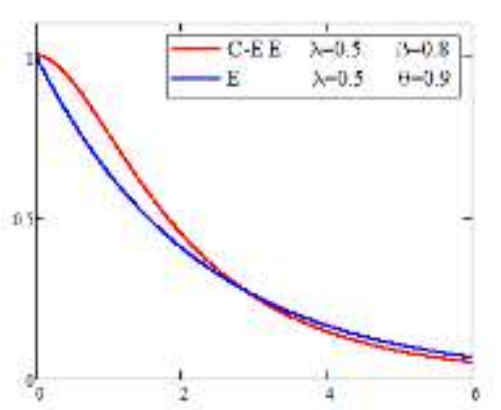

(b)

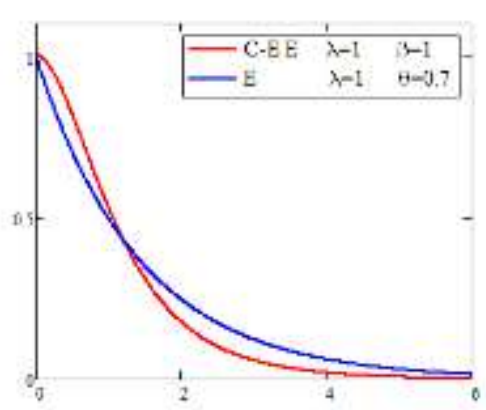

(c)

Figure 1. (a), (b) and (c) The $\bar{F}(x)$ and $\bar{G}(\theta x)$ at different parameters values.

Figure 1 (a), (b) and (c) show the single cross property visually for different values of the unknown parameters.

ii Let $\lambda=1, \beta=3$ and $\theta=0.9385107$, then $F$ and $G$ have the same mean, so a single crossing does occur, and $F$ has smaller variance than $G$. Figure 2 below shows this property visually for a given values for the unknown parameters.

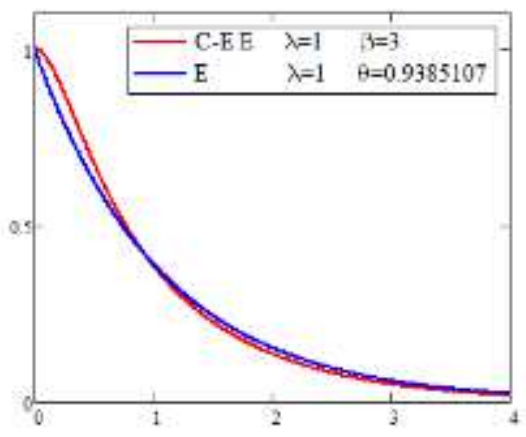

Figure 2. The $\bar{F}$ and $\bar{G}$ at $\lambda=1, \beta=3$ and $\theta=0.9385107$. 
At $\theta=0.9385107, \beta=3$ and $\lambda=1$, the variance of $G$ is 1.032239 , while the variance of $F$ at same values is 0.6280421 . It's clearly that $F$ have smaller variance than $G$.

iii $\mathrm{C}-E E$ is an IFRA.

The cumulative hazard rate functions of the composed- exponential exponential $(\mathrm{C}-E E)$ is given by

$$
H(x)=-\ln \bar{F}(x)=\lambda x\left(1-e^{-\beta x}\right),
$$

where $\bar{F}(x)$ is the survival function of $\mathrm{C}-E E$ distribution. Then,

$$
\frac{H(x)}{x}=\frac{\lambda x\left(1-e^{-\beta x}\right)}{x}=\lambda\left(1-e^{-\beta x}\right),
$$

for $\lambda, \beta>0$ the quantity $e^{-\beta x}$ controls the behavior of the function $\frac{H(x)}{x}$, while $e^{-\beta x}$ is a decreasing function, so $1-e^{-\beta x}$ is an increasing function, then we can conclude that $F$ is IFRA.

The rest of the paper is outlined as follows. In Section 2, special families and distributions are derived from the proposed generator. The statistical properties include quantile functions, moments and incomplete moments are derived in Section 3. Probability weighted moments, the order statistics and their moments are investigated in Sections 4 and 5. Section 6 disscusses the entropies of the propesed generators. The reliability properties include survival function, hazard and cumulative hazard functions and also mean residual and mean reversed life functions are derived in Section 7. In Section 8 , We discuss the method of likelihood estimation to derive the equations used for estimating the unknown parameters. To examine the performance of the new generator section 9 gives a smiulation of one generated model from the generator and compare the performance of the produced model against different models.

\section{New Families}

In this section, we introduce new families that can be used to produce a wide range of new useful distributions. Such families are listed below.

\subsection{The composed exponential-generated family}

Suppose $G(x)=1-e^{-\lambda x},(\lambda, x>0)$ is the cdf of the exponential distribution, a new exponential family can be introduced using (1). This family will be named the composedexponential $Q$ family $(C-$ exponential $Q(C-E Q))$, and its cdf is given by

$$
F(x)=1-e^{-\lambda x \cdot Q(x)},
$$

with corresponding pdf

$$
f(x)=\lambda e^{-\lambda x \cdot Q(x)}[x \cdot q(x)+Q(x)] .
$$

The following models are derived directly from the family, in (4).

\subsubsection{The composed exponential-exponential distribution.}

Inserting $Q(x)=1-e^{-\beta x}$ (for $\beta, x>0$ ) as the cdf of the exponential distribution in (4). The composed-exponential exponential $(C-E E)$ distribution with cdf $F(x)=1-e^{-\lambda x \cdot\left[1-e^{-\beta x}\right]}$, results. 


\subsubsection{The composed exponential-Weibull distribution.}

Inserting $Q(x)=1-e^{-\left(\frac{x}{\alpha}\right)^{\beta}}$ (for $\beta, \alpha, x>0$ ) as the cdf of the Weibull distribution in (4). The composed-exponential Weibull $(C-E W)$ distribution with cdf

$F(x)=1-e^{-\lambda x \cdot\left[1-e^{-\left(\frac{x}{\alpha}\right)^{\beta}}\right]}$, results.

\subsection{The composed Lomax-generated family}

Suppose $G(x)=1-(1+\gamma x)^{-\theta},(\gamma, \theta, x>0)$ is the cdf of the Lomax distribution, a new Lomax family can be introduced using (1). This family will be named the composedLomax $Q$ family $(C-\operatorname{Lomax} Q(C-E Q))$, and its cdf is given by

$$
F(x)=1-[1+\gamma x \cdot Q(x)]^{-\theta},
$$

with corresponding pdf

$$
f(x)=\theta \gamma[1+\gamma x \cdot Q(x)]^{-\theta-1}[x \cdot q(x)+Q(x)] .
$$

The following models are derived directly from the family, in (5).

\subsubsection{The composed Lomax-exponential distribution.}

Inserting $Q(x)=1-e^{-\lambda x}$ (for $\lambda, x>0$ ) in (5). The composed-Lomax exponential $(C-L E)$ distribution with cdf

$F(x)=1-\left[1+\gamma x \cdot\left(1-e^{-\lambda x}\right)\right]^{-\theta}$, results.

\subsubsection{The composed Lomax-Weibull distribution.}

Inserting $Q(x)=1-e^{-\left(\frac{x}{\alpha}\right)^{\beta}}$ (for $\beta, \alpha, x>0$ ) in (5). The composed-Lomax Weibull $(C-L W)$ distribution with cdf

$$
F(x)=1-\left[1+\gamma x \cdot\left(1-e^{-\left(\frac{x}{\alpha}\right)^{\beta}}\right)\right]^{-\theta}
$$

and corresponding pdf

$$
f(x)=\theta \gamma\left[1+\gamma x \cdot\left(1-e^{-\left(\frac{x}{\alpha}\right)^{\beta}}\right)\right]^{-\theta-1}\left[1+\left[\beta\left(\frac{x}{\alpha}\right)^{\beta}-1\right] e^{-\left(\frac{x}{\alpha}\right)^{\beta}}\right]
$$

results.

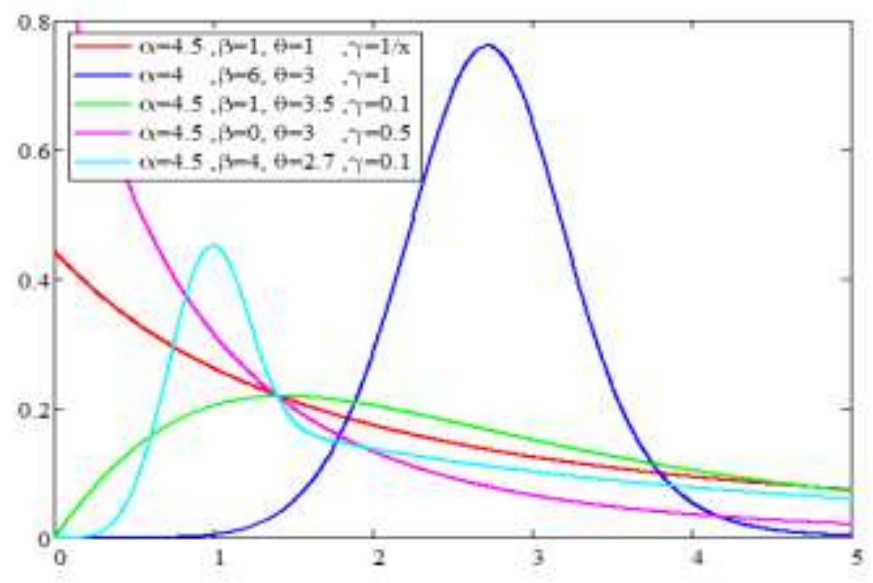

Figure 3. The pdf of the $C-L W$ distribution at different parameter values. 
Figure 3 provide some shapes of the $C-L W$ density curves for selected values of the unknown parameters. The figure shows different shapes of the pdf.

\subsection{Other new families}

Every time one selects a different base distribution, new families arise as a result. The following illustrates our ideas:

Selecting $G(x)$ as a Weibull, log-logistic, Pareto, Burr or Extreme value distribution, the composed Weibull, composed log-logistic, composed Pareto, composed Burr or composed Extreme value-generated families arises.

A lot of families can also be derived and a wide number of distributions could be derived based on this families.

\section{Statistical Properties}

This section explains the statistical properties of the new family in general terms. We then focus on the composed Lomax-Weibull distribution given by (7). Among the statistical properties considered are: the quantiles, the non-central moments and the incomplete moments.

\subsection{Quantiles of the distribution}

The $p^{\text {th }}$ quantile, $x_{p}$, of the new class is the real solution $F\left(x_{p}\right)=p$, which turns out to be the solution of

$$
\text { x. } Q(x)=G^{-1}(p)
$$

Although (9) has no implicit form, it can be solved numerically.

The $p^{\text {th }}$ quantile, $x_{p}$, of the $C-L W$ distribution is the real solution of the following equation:

\subsection{The moments}

$$
x_{p} \cdot e^{-\left(\frac{x_{p}}{\alpha}\right)^{\beta}}-x_{p}+\frac{1}{\gamma}\left[(1-p)^{\frac{-1}{\theta}}-1\right]=0 .
$$

The $r^{\text {th }}$ non-central moment, $\mu_{r}=E\left(X^{r}\right)=\int_{0}^{\infty} x^{r} f(x) d x$, of the new generator can be formulated as

$$
\mu_{r}=\int_{0}^{\infty} x^{r} g(x \cdot Q(x))[x \cdot q(x)+Q(x)] d x=\int_{0}^{\infty} W_{1}^{r}(u) \cdot g(u) d u,
$$

where $W_{1}(u)$ is the solution for $x$ of the function $u=x . Q(x)$.

While the $r^{\text {th }}$ incomplete moment, $m_{r}(z)=E\left(X^{r} \mid x<z\right)=\int_{0}^{z} x^{r} f(x) d x$, of the new generator is given by

$$
m_{r}(z)=E\left(X^{r} \mid x<z\right)=\int_{0}^{z Q(z)} W_{1}^{r}(u) \cdot g(u) d u .
$$

In particular, the $r^{t h}$ non-central moment $\mu_{r}$ and the incomplete moments $m_{r}(z)$ of the $C-L W$ distribution is given by

$$
\mu_{r}=E\left(X^{r}\right)=\frac{\theta}{\beta} \sum_{i=0}^{\infty} \sum_{j=0}^{\infty} i, j,(\theta+1), \gamma \cdot i, j, \beta, \alpha, r, \text { and }
$$




$$
\begin{aligned}
& m_{r}(z)=\theta \sum_{i=0}^{\infty} \sum_{j=0}^{\infty} \frac{\Gamma(i+\theta+1)}{\Gamma(\theta+1) \cdot i !}(-1)^{i+j} \cdot \gamma^{i+1} \cdot\left(\begin{array}{l}
i \\
j
\end{array}\right) \cdot \alpha^{r+i+1}, \\
& \quad \times\left\{\frac{\beta \cdot \Gamma_{1}\left(\frac{r+i+1}{\beta}+1,(j+1)\left(\frac{z}{\alpha}\right)^{\beta}\right)}{\alpha^{\beta} \cdot(j+1)^{\frac{r+i+\beta+1}{\beta}}}-\frac{\Gamma_{1}\left(\frac{r+i+1}{\beta},(j+1)\left(\frac{z}{\alpha}\right)^{\beta}\right)}{\beta \cdot(j+1)^{\frac{r+i+1}{\beta}}}+\frac{\Gamma_{1}\left(\frac{r+i+1}{\beta}, j\left(\frac{z}{\alpha}\right)^{\beta}\right)}{\beta \cdot j^{\frac{r+i+1}{\beta}}}\right\},
\end{aligned}
$$

where $\Gamma_{1}(a, s)=\int_{0}^{s} w^{a-1} e^{-w} d w, a>0$ is the lower incomplete gamma function, and $i, j,(\theta+1), \gamma$ and $i, j, \beta, \alpha, r$ are constants given by

$$
\underset{a, b, c, d}{A}=\frac{\Gamma(a+c)}{\Gamma(c) \cdot a !}\left(\begin{array}{l}
a \\
b
\end{array}\right)(-1)^{a+b} \cdot d^{a+1},
$$

and

$$
\underset{a, b, e, o, v}{B}=\left\{\frac{1}{b^{\frac{v+a+1}{e}}}+\frac{1}{(b+1)^{\frac{v+a+1}{e}}}\left(\frac{e \cdot(v+a-b)}{o^{e} \cdot(b+1)}-1\right)\right\} o^{v+a+1} \cdot \Gamma\left(\frac{v+a+1}{e}\right) .
$$

\section{Probability weighted moments}

The probability weighted moments (PWMs) method can generally be used for estimating parameters of a distribution whose inverse form cannot be expressed explicitly. We calculate the PWMs of the new class since they can be used to obtain the moments of the class. The PWMs of a random variable $X$ are formally defined by

$$
\tau_{s, r}=E\left[X^{r} F^{s}(x)\right]=\int_{0}^{\infty} x^{r} F^{s}(x) f(x) d x
$$

where $r$ and $s$ are non-negative integers and $F($.$) and f($.$) are the cdf and pdf of the$ random variable $X$. The PWMs of the new class with cdf (1) and pdf (2), are given by

$$
\tau_{s, r}=E\left[X^{r} F^{s}(x)\right]=\int_{0}^{1} W_{2}^{r}(z) \cdot Z^{s} d z
$$

where $W_{2}(z)$ is the solution for $x$ of $z=G(x \cdot Q(x))$. The $r^{\text {th }}$ non-central moment of the new class can be obtained by putting $\mathrm{s}=0$ in (17).

In particular, the probability weighted moments for the $C-L W$ distribution is given by:

$$
\tau_{s, r}=\frac{\theta}{\beta} \sum_{i=0}^{\infty} \sum_{j=0}^{\infty} \sum_{k=0}^{\infty}(-1)^{i}\left(\begin{array}{l}
S \\
j
\end{array}\right) \cdot j, k,(i \theta+\theta+1), \gamma^{\cdot} \cdot \begin{gathered}
A \\
j, k, \beta, \alpha,
\end{gathered},
$$

where $j, k,(i \theta+\theta+1), \gamma$ and $j, k, \beta, \alpha, r$ are constants given by (15) and (16), respectively.

\section{Moment of order Statistics}

Order statistics make their appearance in many areas of statistical theory and practice. Let the random variable $X_{r: n}$ be the $r$-th order statistic $\left(X_{1: n} \leq X_{2: n} \leq \ldots \leq X_{n: n}\right)$ in a sample of size $n$ with pdf denotes by $f_{r: n}(x)$ and cdf denotes by $F_{r: n}(x)$.

The $k$-th moment about zero of the $r$-th order statistic are obtained by using a result in Barakat and Abdelkader (2004) and becomes 


$$
E\left(X_{r: n}^{K}\right)=k \sum_{i=n-r+1}^{\infty}(-1)^{i-n+r-1}\left(\begin{array}{c}
i-1 \\
n-r
\end{array}\right)\left(\begin{array}{c}
n \\
i
\end{array}\right) \times I_{k-1}(x),
$$

where $I_{k-1}(x)=\int_{0}^{\infty} x^{k-1}[1-F(x)]^{i} d x$.

In particular, the $k$-th moment about zero of the $r$-th order statistic for the $C-L W$ distribution is given by:

$$
\begin{aligned}
E\left(X_{r: n}^{K}\right)=\frac{k}{\beta} & \sum_{i=n-r+1}^{\infty} \sum_{j=0}^{\infty} \sum_{h=0}^{\infty}\left(\begin{array}{c}
i-1 \\
n-r
\end{array}\right)\left(\begin{array}{c}
n \\
i
\end{array}\right)\left(\begin{array}{c}
j+\theta i-1 \\
\theta i-1
\end{array}\right)\left(\begin{array}{l}
j \\
h
\end{array}\right)(-1)^{i-n+r+j+h-1} \\
& \times \frac{\gamma^{j} \cdot \alpha^{k+j}}{h^{\frac{k+j}{\beta}}} \cdot \Gamma\left(\frac{k+j}{\beta}\right) .
\end{aligned}
$$

\section{Rényi and Shannon entropies}

The entropy measure of a random variable $X$ with density function $f(x)$ is a measure of variation of the uncertainty. One of the popular entropy measures is the Rényi entropy given by

$$
I_{R}(\eta)=\left(\frac{1}{1-\eta}\right) \log \left[\int_{\mathfrak{R}} f^{\eta}(x) d x\right],
$$

where $\eta>0, \eta \neq 1$.

The Shannon entropy which is defined by $E[-\log f(x)]$, is derived from (21) by $\lim _{\eta \rightarrow 1} I_{R}(\eta)$.

The Rényi entropy for the $C-L W$ distribution is given by

$$
I_{R}(\eta)=\left(\frac{1}{1-\eta}\right) \log \left[\begin{array}{c}
(\theta \gamma)^{\eta} \sum_{i=0}^{\infty} \sum_{j=0}^{\infty} \sum_{k=0}^{\infty} \sum_{l=0}^{\infty}\left(\begin{array}{l}
i \\
j
\end{array}\right)\left(\begin{array}{l}
\eta \\
k
\end{array}\right)\left(\begin{array}{l}
k \\
l
\end{array}\right)\left(\frac{(-1)^{i+j+k+l} \cdot \gamma^{i} \cdot \beta^{i+1} \cdot \alpha^{i+1}}{(j+k)^{\frac{i+l \beta+1}{\beta}}}\right) \\
\times \frac{\Gamma(i+\eta(\theta+1)) \cdot \Gamma\left(\frac{i+l \beta+1}{\beta}\right)}{\Gamma(\eta(\theta+1)) \cdot i !}
\end{array}\right] .
$$

\section{Reliability Analysis}

This section presents the survival function, the hazard rate function, the cumulative hazard rate function, the residual and reversed residual lifetime functions for the new generator and especially for the $C-L W$ distribution.

\subsection{Survival Function}

The new generator is a very flexible generator which can be a useful characterization of lifetime data analysis of a given system. The survival function of the new class is defined as:

$$
\bar{F}(x)=1-G(x \cdot Q(x)), \quad \forall x,
$$

while, the survival function of the $C-L W$ distribution is given by:

$$
\bar{F}(x)=\left[1+\gamma x\left(1-e^{-\left(\frac{x}{\alpha}\right)^{\beta}}\right)\right]^{-\theta}, \theta, \gamma, \alpha, \beta>0, x \geq 0 .
$$




\subsection{Hazard Rate and Cumulative Hazard Rate Functions}

The other characteristic of interest of a random variable is the hazard rate function $h(x)$ which is defined as:

$$
h(x)=\frac{g(x Q(x)) \cdot\{x q(x)+Q(x)\}}{1-G(x \cdot Q(x))}, \quad \forall x,
$$

while, the cumulative hazard rate is given by

$$
H(x)=\int_{0}^{x} h(u) d u=-\ln [1-G(x \cdot Q(x))] .
$$

The hazard rate and the cumulative hazard rate functions of the $C-L W$ distribution are, respectively, given by:

$$
\begin{gathered}
h(x)=\theta \gamma\left[1+\gamma x\left(1-e^{-\left(\frac{x}{\alpha}\right)^{\beta}}\right)\right]^{-1} \cdot\left\{1+\beta\left(\frac{x}{\alpha}\right)^{\beta} e^{-\left(\frac{x}{\alpha}\right)^{\beta}}-e^{-\left(\frac{x}{\alpha}\right)^{\beta}}\right\}, \\
H(x)=\theta \ln \left[1+\gamma x\left(1-e^{-\left(\frac{x}{\alpha}\right)^{\beta}}\right)\right] .
\end{gathered}
$$

Figure 4 below provide some plots of the $C-L W$ hazard rate curves for selected values of $\theta, \gamma, \alpha$ and $\beta$.

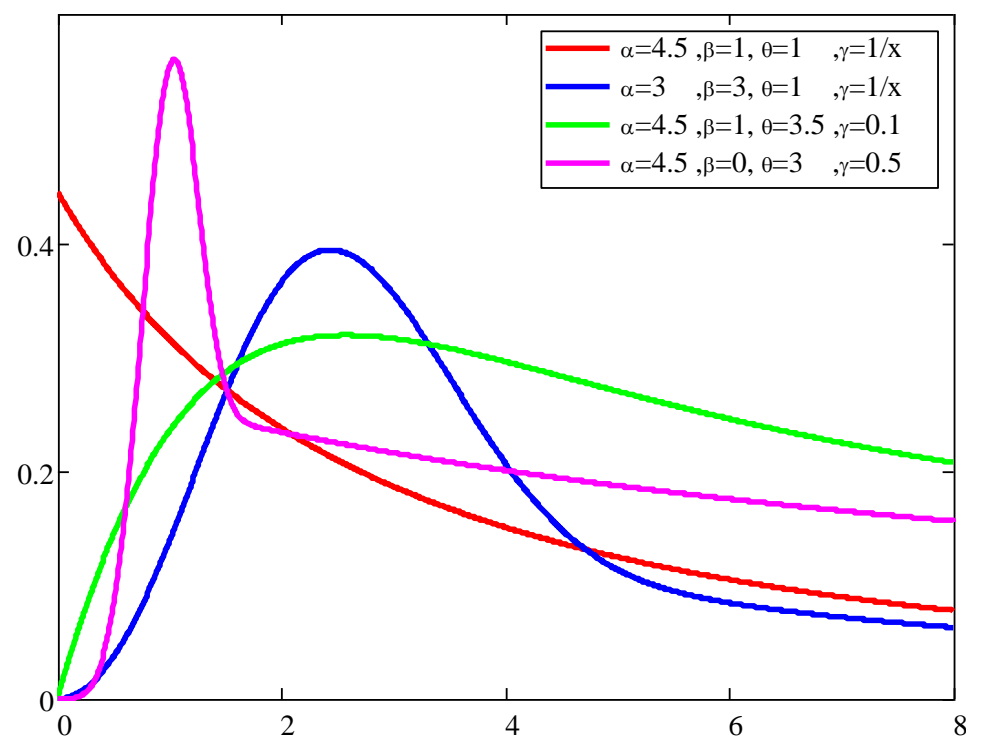

Figure 4. The hazard rate of the composed-Lomax Weibull distribution.

\subsection{Residual life function}

Given that a component survives up to time $y \geq 0$, the residual life is the period beyond $y$ until the time of failure and defined by expectation of the conditional random variable $X \mid X>y$. In reliability, it is well known that the mean residual life function and ratio of two consecutive moments of residual life, determine the distribution uniquely (Gupta and Gupta (1983)). Therefore, we obtain the $r^{\text {th }}$ order moment of the residual life via the general formula

$$
\ddot{m}_{r}(y)=E\left[(X-y)^{r} \mid X>y\right]=\frac{1}{1-F(y)} \int_{y}^{\infty}(X-y)^{r} f(x) d x .
$$

Applying the binomial expansion for $(X-y)^{r}$ and substituting $f(x)$ given by (2) into $\ddot{m}_{r}(y)$, the $r^{\text {th }}$ moment of the residual life of the new family distribution is given by 


$$
\ddot{m}_{r}(y)=\frac{1}{1-F(y)} \sum_{i=0}^{\infty}\left(\begin{array}{l}
r \\
i
\end{array}\right)(-y)^{r-i} \times \int_{y}^{\infty} X^{i} f(x) d x .
$$

An expression for the mean residual lifetime function follows by taking $r=1$.

In particular, the $r^{\text {th }}$ residual life for the $C-L W$ distribution is given by:

$$
\begin{aligned}
& \ddot{m}_{r}(y)=\frac{\theta}{1-F(y)} \sum_{i=0}^{\infty} \sum_{j=0}^{\infty} \sum_{k=0}^{\infty}\left(\begin{array}{l}
r \\
i
\end{array}\right)\left(\begin{array}{l}
j \\
k
\end{array}\right) \frac{\Gamma(j+\theta+1)}{\Gamma(\theta+1) \cdot j !}(-y)^{r-i}(-1)^{j+k} \cdot \gamma^{j+1} \cdot \alpha^{i+j+1} \\
& \quad \times\left\{\frac{\beta \cdot \Gamma_{2}\left(\frac{i+j+1}{\beta}+1,(k+1)\left(\frac{y}{\alpha}\right)^{\beta}\right)}{\alpha^{\beta} \cdot(k+1)^{\frac{i+j+\beta+1}{\beta}}}-\frac{\Gamma_{2}\left(\frac{i+j+1}{\beta},(k+1)\left(\frac{y}{\alpha}\right)^{\beta}\right)}{\beta \cdot(k+1)^{\frac{i+j+\beta+1}{\beta}}}+\frac{\Gamma_{2}\left(\frac{i+j+1}{\beta}, k\left(\frac{y}{\alpha}\right)^{\beta}\right)}{\beta \cdot k^{\frac{i+j+1}{\beta}}}\right\},
\end{aligned}
$$

where $\Gamma_{2}(a, s)=\int_{0}^{s} w^{a-1} e^{-w} d w, a>0$ is the upper incomplete gamma function.

\subsection{Reversed residual life function}

The waiting time since failure is the waiting time elapsed since the failure of an item on condition that this failure had occurred in $[0, y]$. Therefore, we obtain the $r^{\text {th }}$ order moment of the reversed residual life via the general formula

$$
\ddot{M}_{r}(y)=E\left[(y-X)^{r} \mid X<y\right]=\frac{1}{F(y)} \int_{0}^{y}(y-X)^{r} f(x) d x
$$

As doing before, then the $r^{\text {th }}$ moment of the reversed residual life of the new family distribution is given by

$$
\ddot{M}_{r}(y)=\frac{1}{F(y)} \sum_{i=0}^{\infty}\left(\begin{array}{l}
r \\
i
\end{array}\right)(y)^{r-i}(-1)^{i} \times \int_{0}^{y} X^{i} f(x) d x .
$$

An expression for the mean reversed residual lifetime function (or, the mean inactivity time) follows by taking $r=1$.

In particular, the $r^{\text {th }}$ reversed residual life for the $C-L W$ distribution is given by:

$$
\begin{aligned}
& \ddot{M}_{r}(y)=\frac{\theta}{F(y)} \sum_{i=0}^{\infty} \sum_{j=0}^{\infty} \sum_{k=0}^{\infty}\left(\begin{array}{l}
r \\
i
\end{array}\right)\left(\begin{array}{l}
j \\
k
\end{array}\right) \frac{\Gamma(j+\theta+1)}{\Gamma(\theta+1) \cdot j !}(y)^{r-i}(-1)^{i+j+k} \cdot \gamma^{j+1} \cdot \alpha^{i+j+1} \\
& \quad \times\left\{\frac{\beta \cdot \Gamma_{1}\left(\frac{i+j+1}{\beta}+1,(k+1)\left(\frac{y}{\alpha}\right)^{\beta}\right)}{\alpha^{\beta} \cdot(k+1)^{\frac{i+j+\beta+1}{\beta}}}-\frac{\Gamma_{1}\left(\frac{i+j+1}{\beta},(k+1)\left(\frac{y}{\alpha}\right)^{\beta}\right)}{\beta \cdot(k+1)^{\frac{i+j+\beta+1}{\beta}}}+\frac{\Gamma_{1}\left(\frac{i+j+1}{\beta}, k\left(\frac{y}{\alpha}\right)^{\beta}\right)}{\beta \cdot k^{\frac{i+j+1}{\beta}}}\right\} .
\end{aligned}
$$

\section{Estimation}

In this section we introduce the method of likelihood estimation to derive the equations used for estimating the unknown parameters.

Here, we consider estimation of the unknown parameters of the new class by the maximum likelihood method. Let $x_{1}, \ldots, x_{n}$ be a random sample from (2). Let $\boldsymbol{\Theta}$ be a $q \times 1$ vector of the unknown parameter(s) in the proposed class. The log-likelihood function $\mathcal{L}=\log \ell(\Theta)$ is

$$
\mathcal{L}=\sum_{i=1}^{n} \log \left[g\left(x_{i} \cdot Q\left(x_{i}\right)\right)\right]+\sum_{i=1}^{n} \log \left[x_{i} \cdot q\left(x_{i}\right)+Q\left(x_{i}\right)\right]
$$

Then, the first derivative(s) of $\mathcal{L}$ with respect to the vector of the parameter(s) is (are) 


$$
\frac{d \mathcal{L}}{d \Theta}=\sum_{i=1}^{n} \frac{\frac{\partial g\left(x_{i} \cdot Q\left(x_{i}\right)\right)}{\partial \Theta}}{g\left(x_{i} \cdot Q\left(x_{i}\right)\right)}+\sum_{i=1}^{n} \frac{x_{i} \cdot \frac{\partial q\left(x_{i}\right)}{\partial \Theta}+\frac{\partial Q\left(x_{i}\right)}{\partial \Theta}}{x_{i} \cdot q\left(x_{i}\right)+Q\left(x_{i}\right)}
$$

The maximum likelihood estimate(s) (MLE(s)) of $\boldsymbol{\Theta}$, say $\widehat{\boldsymbol{\Theta}}$, is (are) the simultaneous solution(s) of the equation(s) $\frac{\mathrm{d} \mathcal{L}}{\mathrm{d} \Theta}$.

Maximization of (32) can be performed by using well established routines in the $\mathrm{R}$ statistical package.

\section{Application}

In this section, we use simulated data and real data (censored and uncensored) sets to compare the fits of the new model (composed-Lomax Weibull) and illustrate the usefulness of the new model.

\subsection{Simulation study}

To assess the behavior of the maximum likelihood estimators of the parameters $\alpha, \beta, \theta$ and $\gamma$ under the finite samples, we construct a Monte Carlo simulation for the composed Lomax Weibull $(C-L W)$ distribution. All results were obtained from 3000 Monte Carlo replications and the simulations were carried out using the statistical software package $\mathrm{R}$. In each replication a random sample of size $\mathrm{n}$ is drawn from the $C-L W$ distribution. The true parameter values used in the data generating processes are $\alpha=3.5$, $\beta=6.5, \theta=2.8$ and $\gamma=5.2$. Table 1 presents the mean maximum likelihood estimates of the parameters, the bias and the root mean squared errors (RMSE) for different samples of sizes $n=50, n=80$ and $n=100$.

Table 1: Mean estimates, bias and root mean squared errors of $\alpha, \beta, \theta$ and $\gamma$.

\begin{tabular}{c|c|c|c|c}
\hline $\boldsymbol{n}$ & Parameter & Mean estimate & Bias & RMSE \\
\hline \multirow{4}{*}{$\boldsymbol{n}=\mathbf{5 0}$} & $\boldsymbol{\alpha}$ & 3.490231 & 0.00976 & 2.27465 \\
\cline { 2 - 5 } & $\boldsymbol{\beta}$ & 5.437403 & 1.062597 & 2.67904 \\
\cline { 2 - 5 } & $\boldsymbol{\theta}$ & 7.481331 & -4.681331 & 11.92947 \\
\cline { 2 - 5 } & $\boldsymbol{\gamma}$ & 39.65847 & -34.45847 & 598.92578 \\
\hline \multirow{4}{*}{$\boldsymbol{n}=\mathbf{8 0}$} & $\boldsymbol{\alpha}$ & 3.176739 & 0.323261 & 1.60868 \\
\cline { 2 - 5 } & $\boldsymbol{\beta}$ & 6.035708 & 0.464292 & 2.07335 \\
\cline { 2 - 5 } & $\boldsymbol{\theta}$ & 5.315368 & -2.515368 & 8.36179 \\
\hline \multirow{4}{*}{$\boldsymbol{n}=\mathbf{1 0 0}$} & $\boldsymbol{\alpha}$ & 11.02973 & -5.82973 & 103.62223 \\
\cline { 2 - 5 } & $\boldsymbol{\beta}$ & 2.940491 & 0.559509 & 1.45777 \\
\cline { 2 - 5 } & $\boldsymbol{\theta}$ & 5.97697 & 0.52303 & 1.58323 \\
\cline { 2 - 5 } & $\boldsymbol{\gamma}$ & 6.37964 & -1.57964 & 6.50735 \\
\hline
\end{tabular}

Based on table 1 results, we notice that the biases and root mean squared errors of the maximum likelihood estimators of $\alpha, \beta, \gamma$ and $\theta$ decay toward zero as the sample size increases. Also, the bias of the parameter $\alpha$ is increasing; the root mean square error is goes down. 


\subsection{Prices of children's wooden toys - uncensored}

The data is obtained from the Open University (1993). The data represents the prices of 31 different children's wooden toys on sale in a Suffolk craft shop in April 1991.

In order to determine the shape of the most appropriate hazard function for modeling, graphical analysis data may be used. In this context, the total time in test (TTT) plot is very useful (for more details see Aarset (1987)).

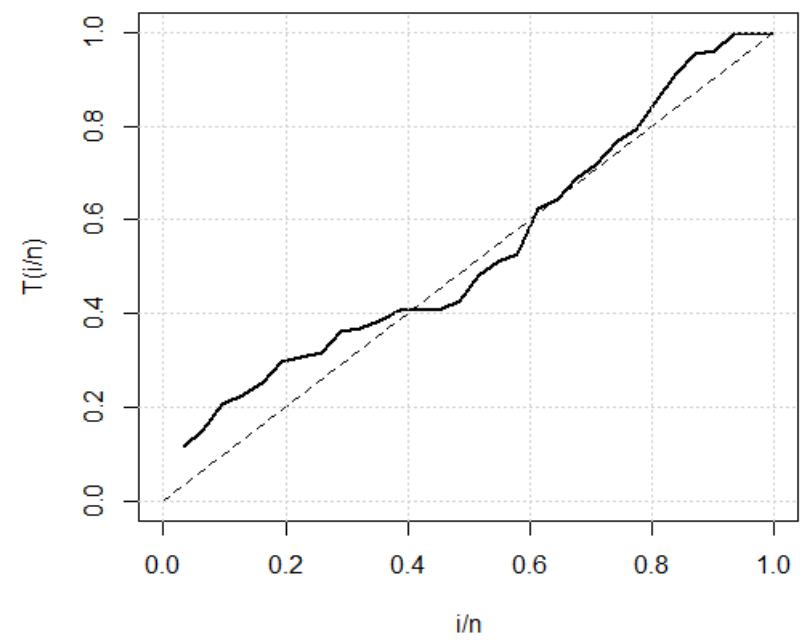

Figure 5. The TTT plot of the children's wooden toys Prices.

The TTT plot for prices of children's wooden toys data is displayed in Figure 5, which provides evidence that a constant hazard rate is adequate.

Table 2. MLEs (standard errors in parentheses) to the children's wooden toys Prices data.

\begin{tabular}{|c|c|c|c|c|c|}
\hline Model & \multicolumn{5}{|c|}{ Estimates } \\
\hline C- L W & $\begin{array}{c}\alpha=1.3554 \\
(0.30207)\end{array}$ & $\begin{array}{c}\beta=2.2289 \\
(0.8299)\end{array}$ & $\begin{array}{c}\gamma=26.2167 \\
(33.6470)\end{array}$ & $\begin{array}{c}\theta=0.00976 \\
(0.0128)\end{array}$ & \\
\hline LW & $\begin{array}{c}\alpha=3.1912 \\
(\mathrm{NaN})\end{array}$ & $\begin{array}{c}\boldsymbol{\beta}=1.5798 \\
(1.2938) \\
\end{array}$ & $\begin{array}{c}\gamma=1.9875 \\
(\mathrm{NaN})\end{array}$ & $\begin{array}{c}\boldsymbol{\theta}=1.7974 \\
(6.3077)\end{array}$ & \\
\hline Kw-LL & $\begin{array}{c}a=16.9977 \\
(25.1455)\end{array}$ & $\begin{array}{c}\boldsymbol{b}=16.4653 \\
(\mathrm{NaN})\end{array}$ & $\begin{array}{c}\alpha=0.08098 \\
(0.2174)\end{array}$ & $\begin{array}{c}\boldsymbol{\beta}=0.4371 \\
(\mathrm{NaN})\end{array}$ & \\
\hline N-MW & $\begin{array}{c}\alpha=0.00152 \\
(\mathrm{NaN})\end{array}$ & $\begin{array}{c}\boldsymbol{\beta}=0.1538 \\
(\mathrm{NaN})\end{array}$ & $\begin{array}{c}\boldsymbol{\gamma}=1.2278 \\
(0.2959)\end{array}$ & $\begin{array}{c}\boldsymbol{\theta}=1.2459 \\
(1.0263) \\
\end{array}$ & $\begin{array}{c}\lambda=1.0 \mathrm{e}-10 \\
(0.0526)\end{array}$ \\
\hline AW & $\begin{array}{l}\alpha=0.1553 \\
(0.057079)\end{array}$ & $\begin{array}{c}\boldsymbol{\beta}=1.0 \mathrm{e}-10 \\
(3.2797 \mathrm{e}-11)\end{array}$ & $\begin{array}{c}\gamma=1.6174 \mathrm{e}-10 \\
(6.2685 \mathrm{e}-11)\end{array}$ & $\begin{array}{c}\boldsymbol{\theta}=1.22797 \\
(0.16996)\end{array}$ & \\
\hline $\mathrm{Kw}-\mathrm{P}$ & $\begin{array}{c}a=3.5761 \\
(2.6832)\end{array}$ & $\begin{array}{c}\boldsymbol{b}=140.0009 \\
(468.6390) \\
\end{array}$ & $\begin{array}{c}\alpha=0.1034 \\
(0.1695) \\
\end{array}$ & $\begin{array}{c}\boldsymbol{\beta}=0.2365 \\
(0.2763)\end{array}$ & \\
\hline Kw-MIW & $\begin{array}{l}a=1.5495 \\
(202.1122) \\
\end{array}$ & $\begin{array}{c}\boldsymbol{b}=1.5744 \\
(0.4095) \\
\end{array}$ & $\begin{array}{c}\alpha=2.8733 \\
(0.7477) \\
\end{array}$ & $\begin{array}{c}\boldsymbol{\theta}=1.7185 \\
(0.000)\end{array}$ & $\begin{array}{l}\lambda=1.7185 \\
(224.1511) \\
\end{array}$ \\
\hline B-NBW & $\alpha=7.9274$ & $\boldsymbol{\beta}=0.9267$ & $\gamma=3.7408$ & $\boldsymbol{\theta}=0.0147$ & $\lambda=1.8061$ \\
\hline
\end{tabular}




\begin{tabular}{c||c|c|c|c|c}
\hline & $(11.8457)$ & $(0.2480)$ & $(6.6691)$ & $(0.0271)$ & $(1.0044)$ \\
\hline \multirow{2}{*}{ W-L } & $\begin{array}{c}\boldsymbol{a}=186.9995 \\
(2684.6007)\end{array}$ & $\begin{array}{c}\boldsymbol{b}=3.48495 \\
(2.9544)\end{array}$ & $\begin{array}{c}\boldsymbol{\alpha}=0.0644 \\
(0.5496)\end{array}$ & $\begin{array}{c}\boldsymbol{\beta}=0.0644 \\
(0.2125)\end{array}$ & \\
\hline \multirow{2}{*}{$\mathrm{EGF}$} & $\boldsymbol{\alpha = 1 7 . 7 4 9 2}$ & $\boldsymbol{\beta}=11.3067$ & $\boldsymbol{\theta}=0.2146$ & $\lambda=56.6383$ & \\
$(18.1465)$ & $(\mathrm{NaN})$ & $(\mathrm{NaN})$ & $(198.3517)$ & \\
\hline \multirow{2}{*}{ ETW } & $\begin{array}{c}\boldsymbol{\alpha}=0.4537 \\
(1.5502)\end{array}$ & $\begin{array}{c}\boldsymbol{\beta}=0.4727 \\
(0.3562)\end{array}$ & $\begin{array}{c}\boldsymbol{\theta}=16.4376 \\
(48.6984)\end{array}$ & $\begin{array}{c}\lambda=0.6095 \\
(0.4843)\end{array}$ & \\
\hline
\end{tabular}

We compare the fitting of the $C-L W$ distribution with 10 non-nested models as the Lomax Weibull (LW), the Kumaraswamy Log-Logistic (Kw-LL), the new modified Weibull (NMW), the additive Weibull (AW), the Kumaraswamy Pareto (Kw-P), the Kumaraswamy modified inverse Weibull (Kw-MIW), the Burr XII Negative Binomial Weibull (B-NBW), the Weibull-Lomax (W-L), the exponentiated generalized Frechet (EGF), the exponentiated transmuted Weibull (ETW) and the Complementary Burr III Poisson (C-BIII-P) introduced by Cordeiro (2014), de Santana et al. (2012), Almalki and Yuan (2013), Xie and Lai (1996), Bourguignon et al. (2013), Aryal and Elbatal (2015), Ramos (2015), Tahir et al. (2015), Cordeiro et al. (2013) and Hassan et al. (2015) respectively.

In each case, the parameters are estimated by maximum likelihood and also model selection is carried out using Akaike information criterion (AIC), consistent Akaike information criterion (CAIC), Hannan-Quinn information criterion (HQIC), Bayesian information criterion (BIC), Anderson-Darling $\left(\mathrm{A}^{*}\right)$ and Cram'er-von Mises $\left(\mathrm{W}^{*}\right)$ to compare the fitted models.

The calculation carried out using the $\mathrm{R}$ code (AdequacyModel). In general, the smaller the values of these statistics, the better the fit to the data.

The estimates of the parameters and the standard error values of this estimates are listed in Table 2 while Table 3, gives the rest of the statistics as AIC, CAIC, BIC, HQIC, W*, $\mathrm{A}^{*}$ and $\mathrm{K}-\mathrm{S}$ values. 
Table 3. The measures AIC, CAIC, BIC, HQIC, $\mathbf{W}^{*}, A^{*}$ and $\mathrm{K}-\mathrm{S}$ (p-value in parentheses) to the children's wooden toys Prices data.

\begin{tabular}{c||c|c|c|c|c|c|c}
\hline Model & AIC & CAIC & BIC & HQIC & $\boldsymbol{W}^{*}$ & $\boldsymbol{A}^{*}$ & $\mathbf{K - S}$ \\
\hline $\begin{array}{c}\text { C- L } \\
\text { W }\end{array}$ & $\mathbf{1 5 2 . 3 6 1 9}$ & $\mathbf{1 5 3 . 9 0 0 3}$ & $\mathbf{1 5 8 . 0 9 7 8}$ & $\mathbf{1 5 4 . 2 3 1 6}$ & $\mathbf{0 . 0 2 9 0 8 9}$ & $\mathbf{0 . 2 2 9 0 7}$ & $\begin{array}{c}\mathbf{0 . 0 8 3 3 1 3} \\
\mathbf{0 . 9 8 2 5})\end{array}$ \\
\hline LW & 157.4773 & 159.0157 & 163.2132 & 159.347 & 0.094721 & 0.57924 & $\begin{array}{c}0.13499, \\
(0.6244)\end{array}$ \\
\hline $\begin{array}{c}\text { KW- } \\
\text { LL }\end{array}$ & 155.4864 & 157.0248 & 161.2223 & 157.3562 & 0.07156 & 0.44536 & $\begin{array}{c}0.12823, \\
(0.6879)\end{array}$ \\
\hline $\begin{array}{c}\text { N- } \\
\text { MW }\end{array}$ & 159.5774 & 161.9774 & 166.7474 & 161.9147 & 0.12739 & 0.76718 & $\begin{array}{c}0.15584, \\
(0.4389)\end{array}$ \\
\hline AW & 157.5774 & 159.1159 & 163.3134 & 159.4472 & 0.12739 & 0.76717 & $\begin{array}{c}0.15583, \\
(0.4389)\end{array}$ \\
\hline KW-P & 154.5486 & 156.0871 & 160.2845 & 156.4184 & 0.048893 & 0.33305 & $\begin{array}{c}0.10497, \\
(0.8842)\end{array}$ \\
\hline $\begin{array}{c}\text { KW- } \\
\text { MIW }\end{array}$ & 158.6616 & 161.0616 & 165.8316 & 160.9989 & 0.047277 & 0.36561 & $\begin{array}{c}0.097263, \\
(0.9311)\end{array}$ \\
\hline $\begin{array}{c}\text { B- } \\
\text { NBW }\end{array}$ & 160.1915 & 162.5915 & 167.3614 & 162.5287 & 0.083676 & 0.52366 & $\begin{array}{c}0.12255, \\
(0.7404)\end{array}$ \\
\hline W-L & 155.3531 & 156.8916 & 161.0891 & 157.2229 & 0.080659 & 0.49293 & $\begin{array}{c}0.13592, \\
(0.6158)\end{array}$ \\
\hline EGF & 155.695 & 155.695 & 161.4309 & 157.5647 & 0.049869 & 0.34970 & $\begin{array}{c}0.092103, \\
(0.9552)\end{array}$ \\
\hline ETW & 154.8948 & 156.4333 & 160.6307 & 156.7646 & 0.055789 & 0.36446 & $\begin{array}{c}0.10849, \\
(0.8589)\end{array}$ \\
\hline
\end{tabular}

Table 3 shows that $\mathrm{C}-L W$ distribution fitted the data better than the other models.

In order to assess if the model is appropriate, we plot in Figure 6 (a) and (b) the histogram of the data and the C- L W, LW, Kw-LL, N-MW, AW and Kw-P distributions and the empirical and their estimated cdf functions, respectively. These plots indicate that the $\mathrm{C}-L W$ distribution provides a better fit to these data than all other competitive lifetime models.

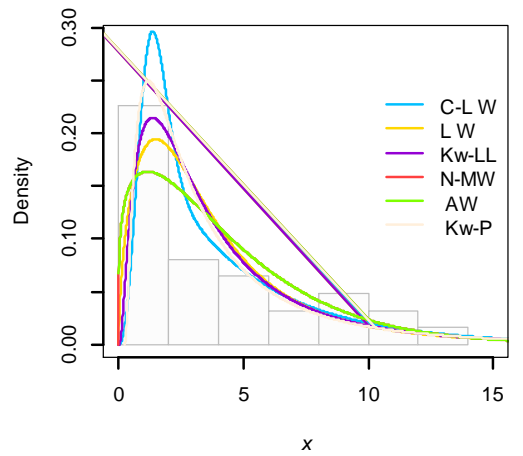

(a)

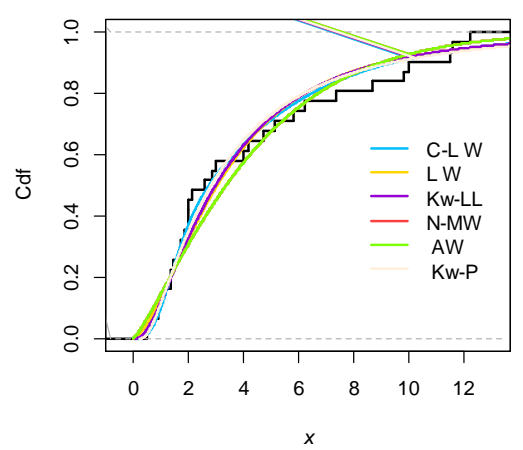

(b) 
Figure 6. (a) Estimated densities of the C- L W, LW, Kw-LL, N-MW, AW and Kw-P distributions for the data. (b) Estimated cdf function from the fitted C- L W, LW, KwLL, N-MW, AW and Kw-P distributions and the empirical cdf for the data.

\subsection{Waiting times before service - uncensored}

The data were reported in Merovci and Elbatal (2013). The data set represents the waiting times (in minutes) before service of 100 bank customers.

Figure 7 below shows the TTT plot of the waiting times before service data

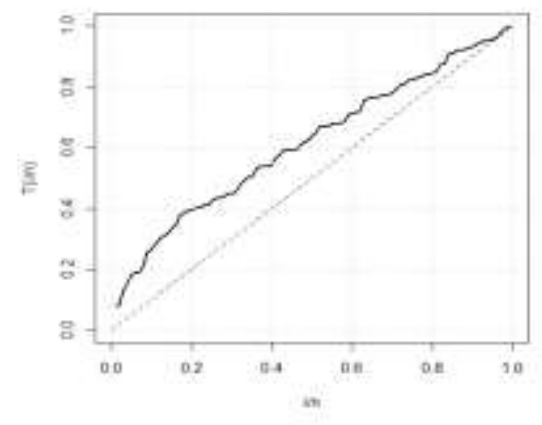

Figure 7. The TTT plot of the waiting times before service data.

The TTT plot for the current data is displayed in Figure 7, which is concave and according to Aarset (1987) provides evidence that the monotonic hazard rate is adequate.

We compare the fitting of the $C-L W$ model with 7 non-nested models. In each case, the parameters are estimated by maximum likelihood and also model selection is carried out using AIC, CAIC, HQIC, BIC, $\mathrm{A}^{*}$ and $\mathrm{W}^{*}$ to compare the fitted models. In general, the smaller the values of these statistics, the better the fit to the data. The estimates of the parameters and the numerical values of the statistics are listed in Table 4 while Table 5 gives the rest of the statistics as AIC, CAIC, BIC, HQIC, $\mathrm{W}^{*}, \mathrm{~A}^{*}$ and $\mathrm{K}-\mathrm{S}$ values.

Table 4. MLEs (standard errors in parentheses) to the waiting times before service data.

\begin{tabular}{c||c|c|c|c|c}
\hline \multicolumn{1}{c||}{ Model } & \multicolumn{5}{c}{ Estimates } \\
\hline \hline \multirow{2}{*}{ C- L W } & $\begin{array}{c}\boldsymbol{\alpha}=\mathbf{8 . 5 7 4 5} \\
(\mathbf{3 . 9 0 6 7})\end{array}$ & $\begin{array}{c}\boldsymbol{\beta}=\mathbf{0 . 9 7 9 5} \\
(\mathbf{0 . 2 8 7 1})\end{array}$ & $\begin{array}{c}\boldsymbol{\gamma}=\mathbf{0 . 0 0 7 7} \\
(\mathbf{0 . 0 0 4 3})\end{array}$ & $\begin{array}{c}\boldsymbol{\theta}=\mathbf{1 8 . 7 0 2 2} \\
(\mathbf{1 0 . 0 4 4 8})\end{array}$ & \\
\cline { 2 - 7 } $\mathrm{LW}$ & $\begin{array}{c}\boldsymbol{\alpha}=6.7942 \\
(108.827)\end{array}$ & $\begin{array}{c}\boldsymbol{\beta}=1.7699 \\
(0.2599)\end{array}$ & $\begin{array}{c}\boldsymbol{\gamma}=5.3959 \\
(152.9615)\end{array}$ & $\begin{array}{c}\boldsymbol{\theta}=3.0217 \\
(2.3701)\end{array}$ & \\
\hline \multirow{2}{*}{$\mathrm{N}-\mathrm{MW}$} & $\begin{array}{c}\boldsymbol{\alpha}=1.0 \mathrm{e}-10 \\
(1.025 \mathrm{e}-10)\end{array}$ & $\begin{array}{c}\boldsymbol{\beta}=0.0306 \\
(0.01185)\end{array}$ & $\begin{array}{c}\boldsymbol{\gamma}=1.4573 \\
(0.2177)\end{array}$ & $\begin{array}{c}\boldsymbol{\theta}=0.1535 \\
(\mathrm{NaN})\end{array}$ & $\begin{array}{c}\lambda=1.0 \mathrm{e}-10 \\
(0.0158)\end{array}$ \\
\hline \multirow{2}{*}{$\mathrm{AW}$} & $\begin{array}{c}\boldsymbol{\alpha}=0.0305 \\
(.00945)\end{array}$ & $\begin{array}{c}\boldsymbol{\beta}=1.0 \mathrm{e}-10 \\
(2.33 \mathrm{e}-06)\end{array}$ & $\begin{array}{c}\boldsymbol{\gamma}=0.0083 \\
(\mathrm{NaN})\end{array}$ & $\begin{array}{c}\boldsymbol{\theta}=1.4579 \\
(0.10859)\end{array}$ & \\
\hline \multirow{2}{*}{$\mathrm{KW}-\mathrm{P}$} & $\begin{array}{c}\boldsymbol{a}=40.4976 \\
(23.9182)\end{array}$ & $\begin{array}{c}\boldsymbol{b}=30.0942 \\
(26.3545)\end{array}$ & $\begin{array}{c}\boldsymbol{\alpha}=0.3768 \\
(0.09999)\end{array}$ & $\begin{array}{c}0.0126 \\
(0.0081)\end{array}$ & \\
\hline \multirow{2}{*}{$\mathrm{KW}-\mathrm{MIW}$} & $\begin{array}{c}\boldsymbol{a}=2.1361 \\
(139.9105)\end{array}$ & $\begin{array}{c}\boldsymbol{b}=1.8659 \\
(0.2923)\end{array}$ & $\begin{array}{c}\boldsymbol{\alpha}=6.1746 \\
(227.055)\end{array}$ & $\begin{array}{c}\boldsymbol{\theta}=1.0 \mathrm{e}-10 \\
(1.22 \mathrm{e}-06)\end{array}$ & $\begin{array}{c}\lambda=3.6762 \\
(240.7804)\end{array}$ \\
\hline
\end{tabular}




\begin{tabular}{c||c|c|c|c|c}
\hline B-NBW & $\begin{array}{c}\boldsymbol{\alpha}=1.0 \mathrm{e}-10 \\
(\mathrm{NaN})\end{array}$ & $\begin{array}{c}\boldsymbol{\beta}=0.6781 \\
(\mathrm{NaN})\end{array}$ & $\begin{array}{c}\boldsymbol{\gamma}=24.5335 \\
(17.4662)\end{array}$ & $\begin{array}{c}\boldsymbol{\theta}=3.7031 \\
(3.6619)\end{array}$ & $\begin{array}{c}\lambda=1.8958 \\
(0.2868)\end{array}$ \\
\hline C-BIII P & $\begin{array}{c}\gamma=1.5892 \\
(0.1070)\end{array}$ & $\begin{array}{c}\boldsymbol{\theta}=4.3694 \\
(1.5062)\end{array}$ & $\begin{array}{c}\lambda=4.1032 \\
(1.2527)\end{array}$ & & \\
\hline
\end{tabular}

Table 5. The measures AIC, CAIC, BIC, HQIC, $\mathbf{W}^{*}, A^{*}$ and $\mathrm{K}-\mathrm{S}$ (p-value in parentheses) to the waiting times before service data.

\begin{tabular}{|c|c|c|c|c|c|c|c|}
\hline Model & $\overline{\mathrm{AIC}}$ & $\overline{\text { CAIC }}$ & BIC & HQIC & $W^{*}$ & $A^{*}$ & $\overline{K-S}$ \\
\hline C- L W & 642.5777 & 642.9987 & 652.9984 & 646.7951 & 0.02041 & 0.1402 & $\begin{array}{l}0.039178, \\
(0.9979) \\
\end{array}$ \\
\hline L W & 643.0161 & 643.4372 & 653.4368 & 647.2336 & 0.02584 & 0.1686 & $\begin{array}{c}0.044112, \\
(0.99) \\
\end{array}$ \\
\hline N-MW & 647.4615 & 648.0998 & 660.4873 & 652.7333 & 0.06283 & 0.3956 & $\begin{array}{l}0.057455, \\
(0.8961) \\
\end{array}$ \\
\hline AW & 645.4614 & 645.8825 & 655.8821 & 649.6788 & 0.06286 & 0.3958 & $\begin{array}{l}0.05779, \\
(0.8921)\end{array}$ \\
\hline $\mathrm{Kw}-\mathrm{P}$ & 644.6283 & 645.0493 & 655.049 & 648.8457 & 0.03698 & 0.2901 & $\begin{array}{c}0.048295, \\
(0.9738) \\
\end{array}$ \\
\hline $\begin{array}{l}\text { KW- } \\
\text { MIW }\end{array}$ & 668.03 & 668.6683 & 681.0559 & 681.0559 & 0.27143 & 1.8249 & $\begin{array}{l}0.10749 \\
(0.1982)\end{array}$ \\
\hline B-NBW & 644.5723 & 645.2106 & 657.5981 & 649.8441 & 0.02126 & 0.1439 & $\begin{array}{c}0.050409 \\
(0.9613) \\
\end{array}$ \\
\hline C-BIII P & 654.7802 & 655.0302 & 662.5957 & 657.9432 & 0.3726 & 2.4415 & $\begin{array}{c}0.46779, \\
(<2.2 \mathrm{e}-16)\end{array}$ \\
\hline
\end{tabular}

Table 5 shows that $C-L W$ distribution fitted the data better than the other models.

In order to assess if the model is appropriate, we plot in Figure 8 (a) and (b) the histogram of the data and the $C-L W$, LW, N-MW, AW, Kw-P and Kw-MIW distributions and the empirical and their estimated cdf functions, respectively. These plots indicate that the Kw-Ps distribution provides a better fit to these data than all other competitive lifetime models.

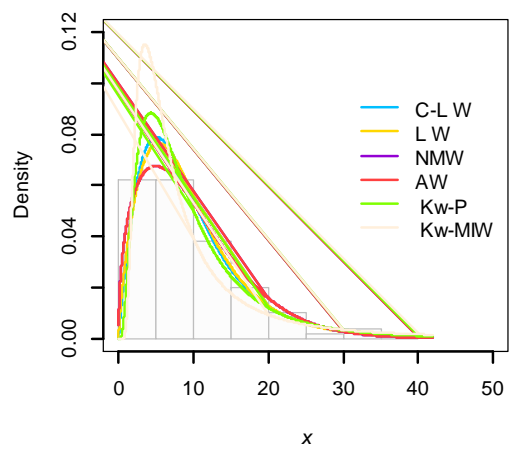

(a)

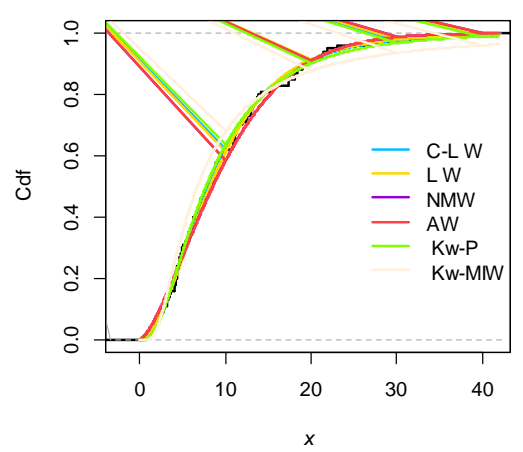

(b) 
Figure 8. (a) Estimated densities of the C- L W, LW, N-MW, AW, Kw-P and Kw-MIW distributions for the data. (b) Estimated cdf function from the fitted C- L W, LW, N-MW, $\mathrm{AW}, \mathrm{Kw}-\mathrm{P}$ and $\mathrm{Kw}-\mathrm{MIW}$ distributions and the empirical cdf for the data.

\subsection{Leukemia data- censored}

Remission times for patients receiving a particular leukemia therapy. Lawless (1982, page 136) gives the results of a study to investigate the effect of a certain kind of therapy for 20 leukemia patients. After the therapy, patients go into remission for some period of time, the length of which is random. The observed times were $1,1,2,2,2,6,6,6,7,8,9$, $9,10,12,13,14,18,1924,26,29,31+, 42,45+, 50+, 57,60,71+, 85+$, 91 weeks.

The times marked with a + indicate patients who were still in remission at the time that the data were analyzed. These are known as right-censored observations because all that is known about them is that they did not come out of remission up to the given time and, presumably, would have come out at some point in time (to the right) of the observed survival times.

Table 6 gives the MLE estimates of the parameters for different models. 
Table 6. MLEs of Leukemia data.

\begin{tabular}{c||c|c|c|c|c|c|c|c}
\hline \multirow{2}{*}{\begin{tabular}{c} 
Model \\
\cline { 2 - 8 }
\end{tabular}} & $\boldsymbol{a}$ & $\boldsymbol{b}$ & $\boldsymbol{\alpha}$ & $\boldsymbol{\beta}$ & $\boldsymbol{\gamma}$ & $\boldsymbol{\theta}$ & $\boldsymbol{\lambda}$ & \multirow{2}{*}{ LL } \\
\hline \multirow{2}{*}{$\begin{array}{c}\text { C- L } \\
\text { W }\end{array}$} & & & $\mathbf{1 . 4 5 6 9}$ & $\mathbf{2 . 7 5 5 1}$ & $\mathbf{0 . 0 4 2 6}$ & $\mathbf{1 . 3 6 4 5}$ & & $\mathbf{- 1 0 7 . 2 5 2 4}$ \\
\hline LW & & & 4.6589 & 1.2557 & 2.8888 & 0.7597 & & -108.4546 \\
\hline KW-LL & 14.7002 & 14.7002 & 0.0972 & 0.3632 & & & & -108.0923 \\
\hline AW & & & 0.0668 & 0.0000 & 0.5683 & 0.8062 & & -109.2581 \\
\hline KW-P & 15.3899 & 6.5549 & 0.2917 & 0.0168 & & & & -108.0328 \\
\hline W-L & 2.1532 & 2.4110 & 0.1267 & 2.4110 & & & & -108.0715 \\
\hline E-GF & & & 6.1637 & 16.3121 & & 8.3539 & 0.1893 & -108.2878 \\
\hline
\end{tabular}

Figure 9 below shows the estimated distribution function various the empirical cdf with lower and upper confidence interval for the values which shows a great fitting to the data.

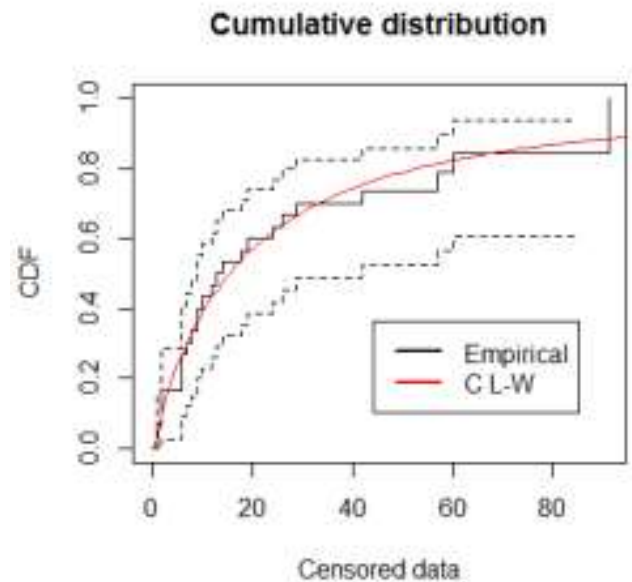

Figure 9. The estimated cdf function from the fitted of new distribution and the empirical cdf for the data with confidence interval of it.

Figure 9, shows that the $C-L W$ line is closely to the data line which indicates a fine fitting to the Leukemia data.

\section{Conclusions}

In this paper, we propose a new method for generating families of continuous distributions, called the composed $-\mathrm{G}$ Q family or shortly ( $-\mathrm{G} Q$ ) family, based on the star-shaped property. Special families and sub-models of the new generator are presented to provides the flexibality of the new generator. The statistical properties shuch as quantiles and moments are disscused in section 3. While, the probability weighted moments, moments of order statistics, and Rényi and Shannon entropies are disscused in sections 4, 5 and 6, respectively. Section 7, presents the reliability properties of the new generator shuch as the survival fuction, the hazard and cumulative hazard functions, moments of residual and reversed life functions. To examine the performance of our new generator and the generated models in fitting several data we use two real sets of data; 
censored and uncensored then comparing the fitting of a new produced model called composed- Lomax Weibull ( $\mathrm{C}-\mathrm{L} \mathrm{W})$ with some well-known models, which provides the best fit to all of the data. A simulation has been performed to assess the behavior of the maximum likelihood estimates of the parameters under the finite samples.

\section{References}

1. Aarset, M. V. (1987). How to identify a bathtub hazard rate. IEEE Transactions on Reliability, 36(1), 106-108.

2. Al-Aqtash, R. (2013). On Generating a New Family of Distributions Using the Logit Function. Ph.D thesis, Central Michigan University Mount Pleasant, Michigan.

3. Alexander, C., Cordeiro, G. M., Ortega, E. M., \& Sarabia, J. M. (2012). Generalized beta-generated distributions. Computational Statistics \& Data Analysis, 56(6), 1880-1897.

4. Alizadeh, M., Emadi, M., Doostparast, M., Cordeiro, G. M., Ortega, E. M., \& Pescim, R. R. (2015). A new family of distributions: the Kumaraswamy odd loglogistic, properties and applications. Hacettepa Journal of Mathematics and Statistics (to appear).

5. Alkarni, S. H. (2013). A Compound Class of Geometric and Lifetimes Distributions. The Open Statistics and Probability Journal, 5, 1-5.

6. Almalki, S. J., \& Yuan, J. (2013). A new modified Weibull distribution. Reliability Engineering \& System Safety, 111, 164-170.

7. Alzaatreh, A., Lee, C., \& Famoye, F. (2013). A new method for generating families of continuous distributions. Metron, 71(1), 63-79.

8. Amini, M., MirMostafaee, S. M. T. K., \& Ahmadi, J. (2014). Log-gammagenerated families of distributions. Statistics, 48(4), 913-932.

9. Aryal, G., \& Elbatal, I. (2015). Kumaraswamy Modified Inverse Weibull Distribution: Theory and Application. Appl. Math, 9(2), 651-660.

10. Barakat, H. M., \& Abdelkader, Y. H. (2004). Computing the moments of order statistics from non-identical random variables. Statistical Methods and applications, 13(1), 15-26.

11. Barlow, R. E., \& Proschan, F. (1981). Statistical theory of reliability and life testing: probability models. FLORIDA STATE UNIV TALLAHASSEE.

12. Bourguignon, M., Silva, R. B., \& Cordeiro, G. M. (2014). The Weibull-G family of probability distributions. Journal of Data Science, 12(1), 53-68.

13. Bourguignon, M., Silva, R. B., Zea, L. M., \& Cordeiro, G. M. (2013). The Kumaraswamy Pareto distribution. Journal of Statistical Theory and Applications, 12(2), 129-144.

14. Cordeiro, G. M., \& da Silva, R. B. (2014). The complementary extended Weibull power series class of distributions. Ciencia \& Natura, 36(3), 1-13.

15. Cordeiro, G. M., \& de Castro, M. (2011). A new family of generalized distributions. Journal of statistical computation and simulation, 81(7), 883-898.

16. Cordeiro, G. M., Alizadeh, M., \& Ortega, E. M. (2014). The exponentiated halflogistic family of distributions: Properties and applications. Journal of Probability and Statistics, 2014.

17. Cordeiro, G. M., Ortega, E. M. M., \& Silva, G. (2012). The beta extended Weibull family. Journal of Probability and Statistical Science, 10, 15-40. 
18. Cordeiro, G. M., Ortega, E. M., \& da Cunha, D. C. (2013). The exponentiated generalized class of distributions. Journal of Data Science, 11(1), 1-27.

19. Cordeiro, G. M., Ortega, E. M., Popović, B. V., \& Pescim, R. R. (2014). The Lomax generator of distributions: Properties, minification process and regression model. Applied Mathematics and Computation, 247, 465-486.

20. de Santana, T. V. F., Ortega, E. M., Cordeiro, G. M., \& Silva, G. O. (2012). The Kumaraswamy log-logistic distribution. Journal of Statistical Theory and Applications, 11(3), 265-291.

21. Ebraheim, A. N. (2014). Exponentiated Transmuted Weibull Distribution: A Generalization of the Weibull Distribution. International Journal of Mathematical, Computational, Physical and Quantum Engineering, 8(6).

22. Eugene, N., Lee, C., \& Famoye, F. (2002). Beta-normal distribution and its applications. Communications in Statistics-Theory and Methods, 31(4), 497-512.

23. Hassan A. S., Abd-Elfattah, A.M. and Mokhtar A. H (2015). The Complementary Burr III Poisson Distribution. Australian Journal of Basic and Applied Sciences. 9(11), 219-228.

24. Lata Gupta, P., \& Gupta, R. C. (1983). On the moments of residual life in reliability and some characterization results. Communications in Statistics-Theory and Methods, 12(4), 449-461.

25. Lawless, J. F. (1982). Statistical methods and model for lifetime data. Wiley\&Sons, New York.

26. Lemonte, A. J., Barreto-Souza, W., \& Cordeiro, G. M. (2013). The exponentiated Kumaraswamy distribution and its log-transform. Brazilian Journal of Probability and Statistics, 27(1), 31-53.

27. Mansour, M. M., \& Mohamed, S. M. (2015). A New Generalized of Transmuted Lindley Distribution. Applied Mathematical Sciences, 9(55), 2729-2748.

28. Marshall, A. W., \& Olkin, I. (1997). A new method for adding a parameter to a family of distributions with application to the exponential and Weibull families. Biometrika, 84(3), 641-652.

29. Merovci, F., \& Elbatal, I. (2013). Transmuted Lindley-geometric distribution and its applications. arXiv preprint arXiv:1309.3774.

30. Nadarajah, S., Teimouri, M., \& Shih, S. H. (2014). Modified beta distributions. Sankhya B, 76(1), 19-48.

31. Pappas, V., Adamidis, K., \& Loukas, S. (2012). A family of lifetime distributions. International Journal of Quality, Statistics, and Reliability, 2012.

32. Pescim, R. R., Cordeiro, G. M., Demétrio, C. G., Ortega, E. M., \& Nadarajah, S. (2012). The new class of Kummer beta generalized distributions. SORT-Statistics and Operations Research Transactions, 36(2), 153-180.

33. Ramos, M. W. A., Percontini, A., Cordeiro, G. M., \& da Silva, R. V. (2015). The Burr XII Negative Binomial Distribution with Applications to Lifetime Data. International Journal of Statistics and Probability, 4(1), p109.

34. Ristić, M. M., \& Balakrishnan, N. (2012). The gamma-exponentiated exponential distribution. Journal of Statistical Computation and Simulation, 82(8), 1191-1206.

35. Santos-Neto, M., Bourguignon, M., Zea, L. M., Nascimento, A. D., \& Cordeiro, G. M. (2014). The Marshall-Olkin extended Weibull family of distributions. Journal of Statistical Distributions and Applications, 1(1), 9. 
36. Shaw, W. T., \& Buckley, I. R. (2009). The alchemy of probability distributions: beyond Gram-Charlier expansions, and a skew-kurtotic-normal distribution from a rank transmutation map. arXiv preprint arXiv:0901.0434.

37. Silva, R. B., Bourguignon, M., Dias, C. R., \& Cordeiro, G. M. (2013). The compound class of extended Weibull power series distributions. Computational Statistics \& Data Analysis, 58, 352-367.

38. Tahir, M. H., Cordeiro, G. M., Alzaatreh, A. Y. M. A. N., Mansoor, M., \& Zubair, M. (2015). The Logistic-X family of distributions and its applications. Commun Stat-Theory Methods (forthcoming).

39. Tahir, M. H., Zubair, M., Cordeiro, G. M., Alzaatreh, A., \& Mansoor, M. (2016). The Poisson-X family of distributions. Journal of Statistical Computation and Simulation, 1-21.

40. Tahmasebi, S., \& Jafari, A. A. (2015). Exponentiated extended Weibull-power series class of distributions. arXiv preprint arXiv:1503.08653.

41. The Open University (1963). MDST242 Statistics in Society Unit A0: Introduction, 2nd ed., Milton Keynes: The Open University

42. Torabi, H., \& Hedesh, N. M. (2012). The gamma-uniform distribution and its applications. Kybernetika, 48(1), 16-30.

43. Xie, M., \& Lai, C. D. (1996). Reliability analysis using an additive Weibull model with bathtub-shaped failure rate function. Reliability Engineering \& System Safety, 52(1), 87-93.

44. Zografos, K., \& Balakrishnan, N. (2009). On families of beta-and generalized gamma-generated distributions and associated inference. Statistical Methodology, 6(4), 344-362. 